\title{
POLYMER NANOCOMPOSITE BASED ON STYRENE WITH BUTYL METHACRYLATE AND INORGANIC SEMICONDUCTOR CdS
}

\author{
Mihail Iovu ${ }^{\mathrm{a}}$, Mihai Enachescu ${ }^{\mathrm{b}}$, Ion Culeac ${ }^{\mathrm{a}^{*}}$, Victor Verlan ${ }^{\mathrm{a}}$, Stefan Robuc, \\ Dionezie Bojin ${ }^{\mathrm{b}}$, Iurie Nistor ${ }^{\mathrm{a}}$, Ion Cojocaru ${ }^{\mathrm{a}}$ \\ ${ }^{a}$ Institute of Applied Physics of Academy of Sciences of Moldova, 5, Academiei str., Chisinau MD 2028, Republic of Moldova \\ ${ }^{b}$ CSSNT, University Politehnica of Bucharest, 313, Splaiul Independentei, sector 6, Bucharest, RO-060042, Romania \\ ${ }^{c}$ Moldova State University, 60, Mateevici str., Chisinau MD 2009, Republic of Moldova \\ e-mail:ionculeac@gmail.com
}

\begin{abstract}
We present experimental results on copolymer-based nanocomposite made of styrene with butyl methacrylate $(1: 1)$ and inorganic semiconductor CdS. Thin film composite samples have been characterized by UVVis absorption and photoluminescent spectroscopy, as well as by transmission electron microscopy. Transmission electron microscope examination confirms a relatively narrow distribution of CdS nanoclusters in the SBMA matrix, which covers the range 2-10 $\mathrm{nm}$. On the other side, the average CdS particles size estimated from the position of first excitonic peak in the UV-Vis absorption spectrum was found to be $2.8 \mathrm{~nm}$ and $4.4 \mathrm{~nm}$ for two samples with different duration of thermal treatment, which is in good agreement with PL experimental data. The PL spectrum for CdS nanocrystals is dominated by near-band-edge emission. The relatively narrow line width (40-45 nm) of the main PL band suggests the nanoparticles having narrow size distribution. On the other side, relatively low PL emission from surface trap states at longer wavelengths were observed in the region 500-750 nm indicating on recombination on defects.
\end{abstract}

Keywords: nanocomposite, polymer matrix, photoluminescence, exciton.

\section{Introduction}

Nanocomposite (NC) materials belong to one of the most dynamic domain of research and development, and they occupy an important place is the field of nanotechnology [1-6]. Nanocomposite materials made of a polymer matrix and an inorganic semiconductor have become a prominent area of research and technology because of their attractive properties [4-6]. NCs offer a variety of new possibilities beyond those of conventional materials as well as compared to NCs constituent components. The basic feature of nanoscale materials resides in the possibility of tuning of their physical and chemical properties through varying the size of incorporated nanoparticles. This actually means obtaining different properties within the same chemical composition just by changing the size of nanoparticles [7-10].

Among existing variety of nanocomposite materials polymer based NCs attract a lot of research efforts because of many advantages, compared to conventional materials. These advantages refer to their relatively simple technology, low cost, easy tuning, etc. Polymer NCs can be obtained in the form of thin films, bulk, or fiber samples, etc., by relatively simple technological methods, among them drop-wise deposition, spin coating, extrusion, etc. Polymer based $\mathrm{NC}$ materials with inorganic semiconductors comprise a polymer material as a matrix and incorporated inorganic semiconductor nanoparticles as fillers [11-12]. The role of the polymer matrix in nanocomposites is to assemble the nanoparticles into clusters, avoiding agglomeration, inducing ordering and orientation in self-assembling structures, etc.

As one of the most important II-VI group semiconductors CdS nanoparticles have received great attention because of their attractive properties and potential for application in photonics and optoelectronics [14-16]. In the present work we report preparation and characterization of photoluminescent polymer-inorganic nanocomposite thin films based on styrene-butylmethacrylate copolymer (SBMA) (1:1) and inorganic semiconductor CdS.

\section{Experimental}

The technology of preparation of the nanocomposite thin films made of styrene with butylmethacrylate (SBMA) (1:1) and inorganic semiconductor $C d S$ was described elsewhere [17-18]. At the first step a solution of cadmium nitrate $\mathrm{Cd}\left(\mathrm{NO}_{3}\right)_{2}$ was mixed with a solution of thiocarbamide, $S C\left(\mathrm{NH}_{2}\right)_{2}$, taken in equimolar ratio. In this way we obtain a complex compound $\mathrm{Cd}\left(\mathrm{NO}_{3}\right)_{2} \times S C\left(\mathrm{NH}_{2}\right)_{2}$, well soluble in distilled water. The SBMA copolymer, which is used as a matrix, was dissolved in an organic solvent, partially hydrophilic (e.g. dimethylformamide). In this way a copolymer solution with concentration $10 \mathrm{~g}$ of polymer in $100 \mathrm{ml}$ solvent was prepared. At the next step an appropriate quantity of $\mathrm{Cd}\left(\mathrm{NO}_{3}\right)_{2} \times S C\left(\mathrm{NH}_{2}\right)_{2}$ related to $\mathrm{CdS}$ concentration of 20 and 40 mass\% relative to SBMA copolymer was added in the solution. All this composition was mixed by vigorous stirring. For preparation of the nanocomposite thin films the final solution was cast onto a clean, flat glass plate, and heated at $100{ }^{\circ} \mathrm{C}$ during $30-60 \mathrm{~min}$.

The composite layers were obtained on clean glass substrates for measuring of optical transmission and photoluminescence. The thickness of the thin film layers was in the range 5-15 $\mu \mathrm{m}$. Nanocomposite thin films were characterized by measuring UV-Vis transmission and photoluminescent (PL) spectra, and by transmission electron microscopy (TEM EM 410). Optical transmission spectra of the samples were registered in the range $400-800 \mathrm{~nm}$ on a 
Specord UV-Vis spectrophotometer or M40 photospectrometer. PL spectra of nanocomposite thin films were measured at room temperature under excitation of a laser beam 337 or $405 \mathrm{~nm}$ using a MDR-23 monochromator and a Hamamatsu photomultiplier module H9319-12 operating in a photon counting regime.

\section{Results and discussion}

Examination of prepared thin films suggests that they contain clusters of CdS of different sizes. This can be seen on nanocomposite thin films $S M B A+40 \% C d S$ deposited on glass substrate (Figure 1). Transmission electron microscope images indicate on a relatively narrow distribution of the CdS nanoclusters in the SBMA matrix, which covers the range $\sim 2-10 \mathrm{~nm}$ (Figure 1a). The small nanoparticle diameter correlates with the strong shift of the PL maximum at $\sim 383 \mathrm{~nm}$. On the other hand, in the case of larger thermal treatment time one can observe a less dispersed but larger nanoclasters diameter (Figure 1b). From the image in Figure $1 \mathrm{~b}$ the nanoparticles size can be estimated to be in the range $\sim 20-30 \mathrm{~nm}$. The UV-Vis absorption spectra of nanocomposite thin-film samples deposited on glass substrates are presented in Figure 2. The position of the main excitonic peak one can clearly distinguish on these spectra. These peaks are situated at $381 \mathrm{~nm}(2.97 \mathrm{eV})$ and $429 \mathrm{~nm}(3.28 \mathrm{eV})$ respectively.

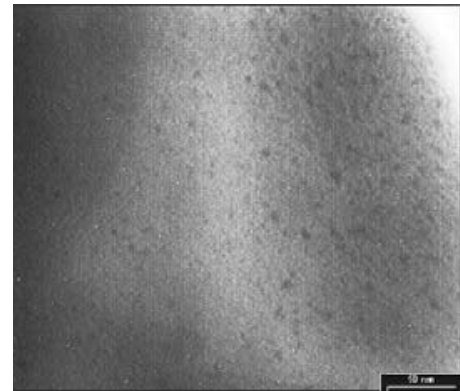

(a)

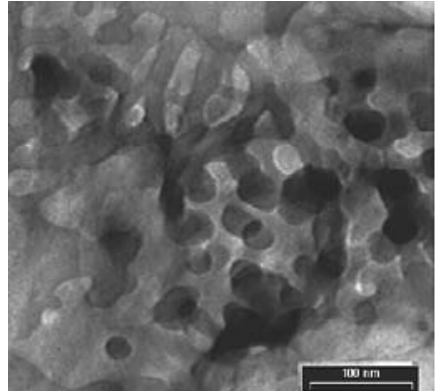

(b)

Figure 1. TEM image of the nanocomposite thin films SBMA+40\%CdS obtained at different thermal treating time 30 (a) and 60 (b) min.

The size of nanoparticles has been evaluated from the position of the first excitonic peak from the data in Figure 2 through the empirical relation [19-21]:

$$
D=\left(-6.6521 \times 10^{-8}\right) \lambda^{3}+\left(1.9557 \times 10^{-4}\right) \lambda^{2}-\left(9.2352 \times 10^{-2}\right) \lambda+(13.29),
$$

where $D(\mathrm{~nm})$ is the diameter of the nanoparticles and $\lambda(\mathrm{nm})$ is the wavelength of the first excitonic peak of the corresponding sample. In this way we can evaluate the diameter of the nanoparticles as $2.8 \mathrm{~nm}$ and $4.4 \mathrm{~nm}$. Such nanoparticles size implies a strong confinement of the charge carriers, while the confinement energies of the electron and hole are much larger than the energy of Coulomb interaction [22,24]. The position of the first excitonic energy can be estimated from the relation, which connects the size of the nanocrystal and the excitonic energy $E$ [21-23]:

$E=E_{g}+\frac{\hbar^{2} \pi^{2}}{2 R^{2}}\left(\frac{1}{m_{e}^{*}}+\frac{1}{m_{h}^{*}}\right)-\frac{1}{R}\left(\frac{1.8 e^{2}}{4 \pi \varepsilon_{0} \varepsilon_{r}}\right)$,

where $E_{g}$ is the energy gap of bulk CdS, $R$ is the size of the nanoparticle, $m_{e}{ }^{*}$ and $m_{h}{ }^{*}$ are the effective masses of the electron and hole. The corresponding values for the effective masses are as reported elsewhere [24-26]: $\frac{m_{e}^{*}}{m_{0}}=0.19$ and $\frac{m_{h}^{*}}{m_{0}}=0.8$; and the dielectric constant $\varepsilon_{r}=5.7 ; \varepsilon_{0}$ is the permittivity of free space and $e$ is the electron charge. If we take the particles diameter as determined from the absorption spectra in Figure 2 as $D_{1}=2.8 \mathrm{~nm}$ and $D_{2}=4.4 \mathrm{~nm}$, then the calculated exciton energies are respectively $2.72 \mathrm{eV}$ and $3.36 \mathrm{eV}$. On the other side the positions of the main excitonic peak determined from the absorption spectra in Figure 2 are $2.97 \mathrm{eV}$ (curve 1) and $3.28 \mathrm{eV}$ (curve 2) respectively. These values are higher than the energy gap $E_{g}$ of the bulk CdS (2.42 eV at $300 \mathrm{~K}$ [24-26]) which indicates on a blue shift of the absorption edge. The increase of the band gap is determined by the quantum size effect of these small crystallites, and the calculated diameter of the nanocrystals $(2.8 \mathrm{~nm}$ and $4.4 \mathrm{~nm})$ is comparative to the excitonic Bohr radius $\sim 3 \mathrm{~nm}$ of CdS [21-23]. 


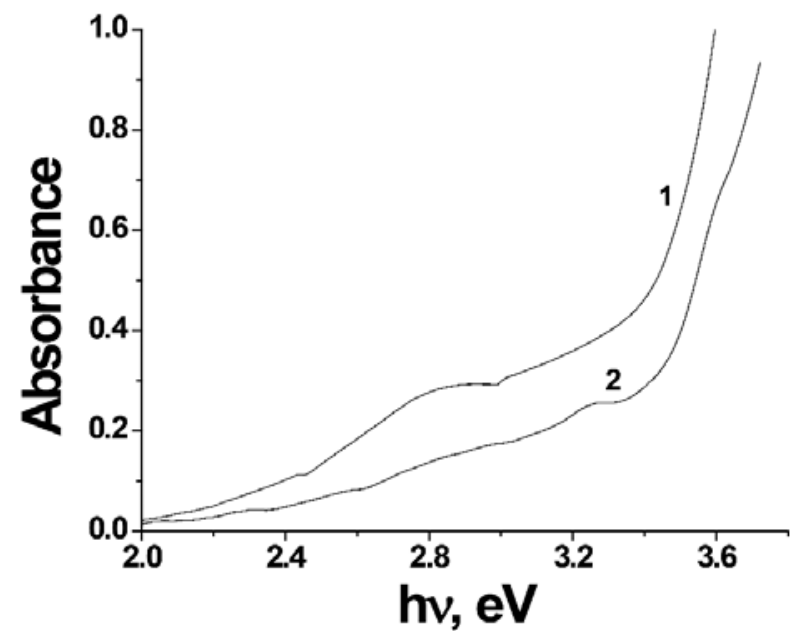

Figure 2. Absorbance spectrum of SBMA+40\%CdS nanocopmposite samples with different nanoparticles size: (1) $2.8 \mathrm{~nm}$ and (2) $4.4 \mathrm{~nm}$.

Figure 3 represents the PL spectra of nanocomposites SBMA/CdS measured in two different samples SBMA/ $\mathrm{CdS}$ characterized by different nanoparticles size [27]. Nanocomposite samples SBMA/CdS exhibit a luminescent signal in the range $350-600 \mathrm{~nm}$, with the position of PL maximums varying in dependence of the dimensions of the $\mathrm{CdS}$ nanoparticles in polymer matrix. Variation of the thermal treatment time leads to variation of the size of CdS nanoparticles and shifts the maximum of the photoluminescence peak in the high energy region. The PL emission peaks of the nanocomposites SBMA/CdS are found to be located at $383 \mathrm{~nm}$ and $463 \mathrm{~nm}$ respectively (samples 2.3 and 2.1 in Figure 3a). The excitonic energy for the corresponding nanocomposite samples (Figure 2) corresponds to 2.97 and 3.28 $\mathrm{eV}$ respectively. These values indicate that the luminescence peaks are Stokes-shifted from their band gap energy. The full width at half maximum (FWHM) of the PL peak in Figure 3 a represents 45 (2.3) and $40 \mathrm{~nm}$ (2.1). The full width of $\sim 40 \mathrm{~nm}$ suggests the size dispersion of the nanoparticles around the mean diameter value is relatively narrow. The broadening of the PL spectra for the sample 2.3 of approximately $\sim 45 \mathrm{~nm}$ can be attributed basically to nanoparticle size dispersion and less to the presence of charges on the surface of the nanoparticles [24-27]. The PL maximums can be attributed to direct transitions from the conduction band to valence band.

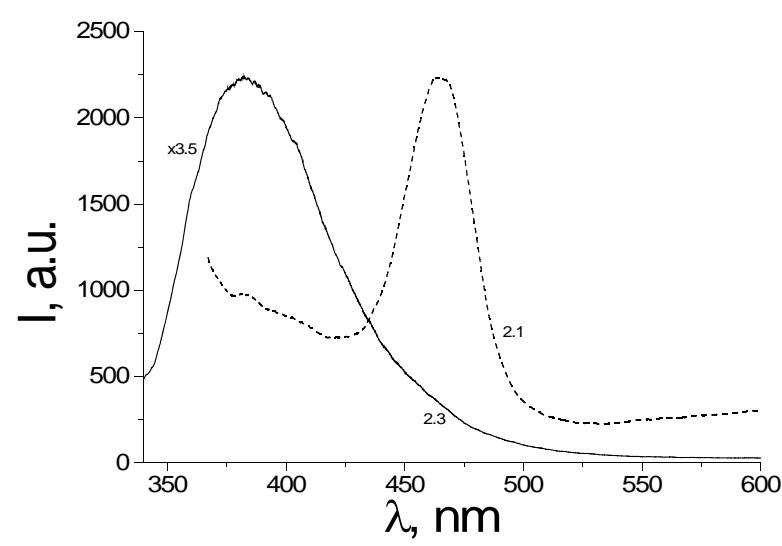

(a)

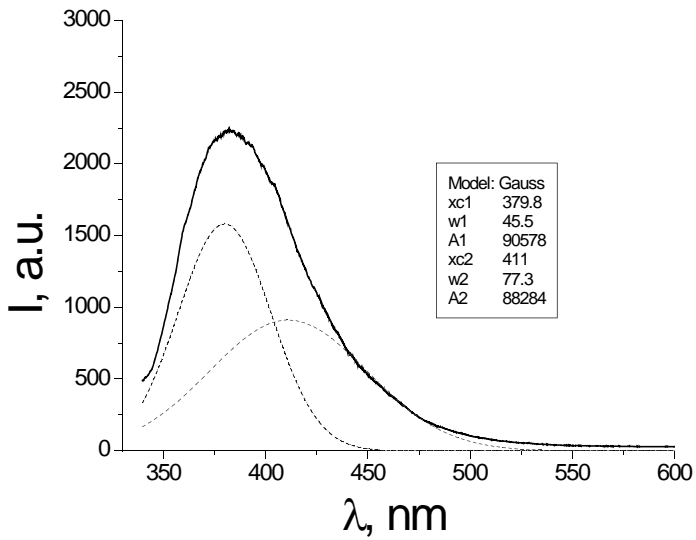

(b)

Figure 3. PL spectra of NC SBMA+40\% CdS measured at room temperature under excitation $337 \mathrm{~nm}$ : (a) sample 2.3 - nanoparticles size $2.4 \mathrm{~nm}$; sample 2.1 - nanoparticles size $4.2 \mathrm{~nm}$;

(b) deconvolution of PL spectra for sample 2.3.

In addition to blue-violet PL main bands nanocomposite samples exhibit several weaker red peaks positioned at $562 \mathrm{~nm}, 599$ and $639 \mathrm{~nm}$ (Figure 4). While the PL maxima in the visible range can be attributed to direct transitions from the conduction band to valence band, the PL emission in the red range can be associated to the transitions from the donor levels to the valence band of CdS [25-27]. 


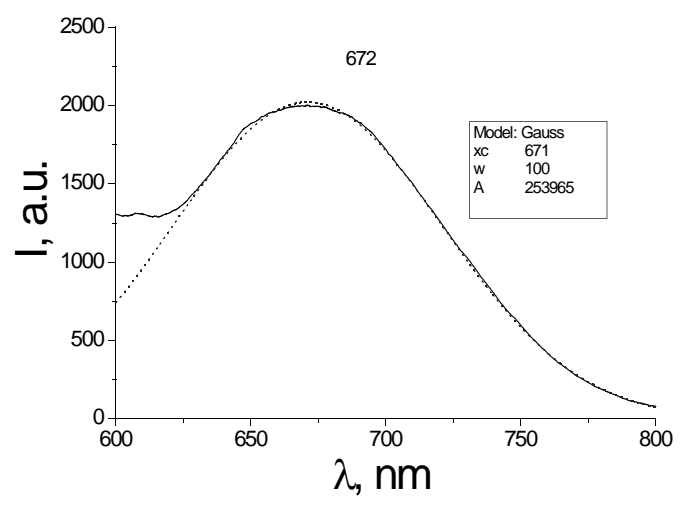

(a)

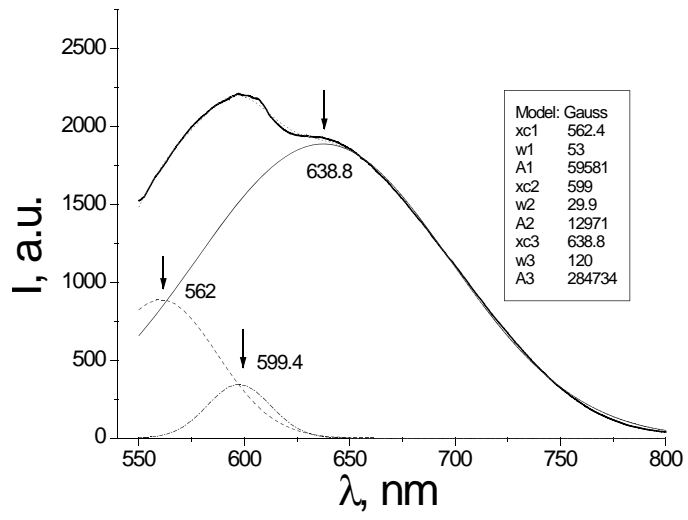

(b)

Figure 4. PL spectra of nanocomposite samples SBMA/CdS under excitation of light beam 405 nm: (a) SBMA+20\%CdS (sample 1.1); (b) SBMA+40\%CdS (sample 2.1).

Figure 5 illustrates the scheme of optical absorption in CdS nanoparticles incorporated in the copolymer matrix SBMA. In the case of confinement the absorption spectrum of the nanocrystallies exhibits a quasi discrete character versus the photon energy and the density of states in quantum dots peaks at certain energies. As a result of the confinement the energetic QDs gap increases compared to bulk semiconductor. The nanocystallite energetic spectrum behaves like an isolate hydrogen atom with the energetic levels $1 s, 1 p, 1 d$, etc.

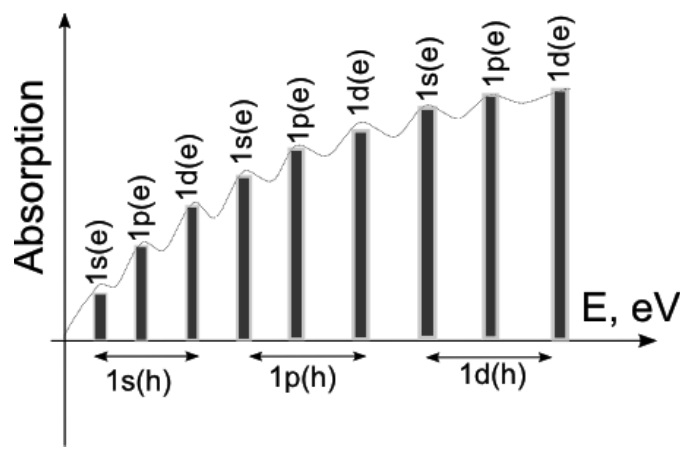

Figure 5. A simplified scheme of QDs energy levels and their absorption bands in the SBMA/CdS nanocomposite.

When a photon is absorbed by the $\mathrm{CdS}$ semiconductor an electron is excited from the valence into the conduction band, and a positively-charged hole is created in the valence band. When the excitation energy corresponds to optical transition $1 s(h)-1 s(e)$ one or two electrons can appear on the energetic level $1 s(e)$ (Figure 5). In the later case the level $1 s(e)$ is split into two sublevels $1_{S 1 / 2}(e)$ and $1_{S 3 / 2}(e)$ with anti-parallel spins. In this way, as a result of optical excitation an exciton is created - a bound state of electron and hole, which are attracted to each other by the electrostatic Coulomb force. The onset of the optical absorption can be considered as the threshold of optical absorption of the CdS nanocrystals. At each absorption event an exciton appears, consequently because of many different energetic levels there are many different groups of excitons. The smallest excitonic energy can be determined from the relation (2), and this energy can be attributed to the absorption threshold of confined nanoparticle.

PL mechanism is illustrated in Figure 6. Under UV radiation the electrons from the holes levels $1 s, 1 p$ and $1 d$ are excited to the electrons levels $1 s, 1 p$ and $1 d$. When the photon energy is absorbed in CdS material excitons appear. In the case of annihilation of these excitons a photon is emitted, according to the transitions $1 s(e)-1 s(h), 1 p(e)-1 s(h)$, $1 d(e)-1 s(h)$, etc. In the case of CdS incorporated in the copolymer SBMA matrix, additional to the transitions described above, there are other transitions with energy transfer from the singlet and triplet levels in the copolymer to the energy levels of CdS nanocrystal $1 s(e), 1 p(e), 1 d(e)$ (Figure 6). Because of the dispersion of the size of CdS nanoparticles in the matrix of SBMA the magnitude of FWHM is a bit larger. Besides, there are PL bands shifted to red, and which can be related to the defects within the bulk of CdS as well as on the surface of nanocrystalites. 


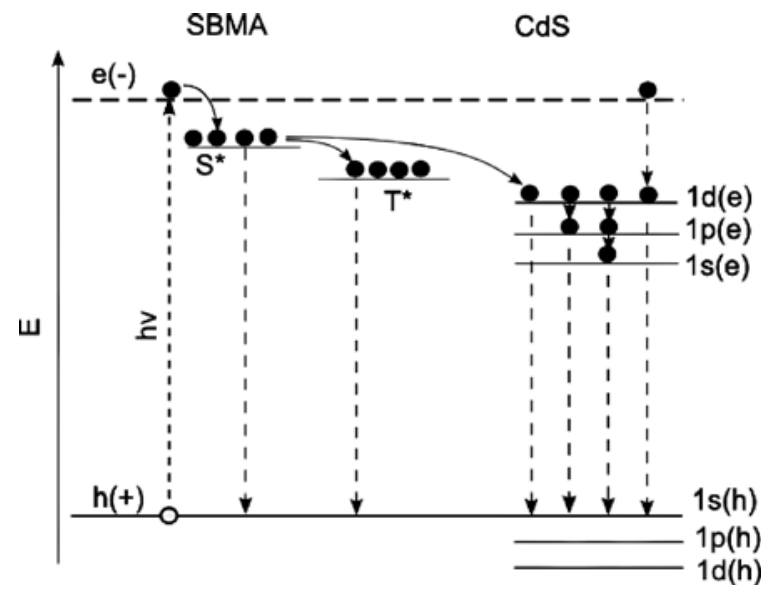

Figure 6. Illustration of the mechanism of energy transfer and photoluminescence in NC.

\section{Conclusions}

Copolymer-based nanocomposites made of styrene with butyl methacrylate and inorganic semiconductor CdS have been investigated. Nanocomposite samples with the concentration of CdS semiconductor 20 and $40 \%$ have been studied. The average CdS particle size estimated from TEM correlates with the particle size estimated from the UV-Vis absorption spectrum and was found to be in the range 2-10 $\mathrm{nm}$. The nanocomposite samples exhibit a major PL band in the visible range $350-500$ and a weak PL signal in the range $600-800 \mathrm{~nm}$.

\section{Acknowledgement}

This research was supported by the Academy of Sciences of Moldova (research grants 11.817.05.03A, 13.820.05.15/RoF and 14.819.02.20A).

\section{References}

1. Paul, D.R.; Robeson, L.M. Polymer nanotechnology: Nanocomposites, Polymer, 2008, 49, pp. 3187-3204.

2. Wang, Y. Fiber-Optic Sensors for Fully-Distributed Physical, Chemical and Biological Measurement. PhD Thesis, Virginia Polytechnic Institute, Blacksburg, Virginia, 2012.

3. Jorge, P.; Martins, M.A.; Trindade, T.; Santos, J.L.; Farahi F. Optical Fiber Sensing Using Quantum Dots, Sensors, 2007, 7, pp. 3489-3534.

4. Li, S.; Lin, M.M.; Toprak, M.S.; Kim, D.K.; Muhammed, M. Nanocomposites of polymer and inorganic nanoparticles for optical and magnetic applications, Nano Reviews, 2010, 1, doi:10.3402/nano.v1i0.5214.

5. Shenhar, R.; Norsten, T.B. Polymer-Mediated Nanoparticle Assembly: Structural Control and Applications, Advanced Materials, 2005, 17, pp. 657-669.

6. Godovsky, D.Y. Device applications of polymer-nanocomposites. Advances in Polymer Sciences, Springer-Verlag, Berlin Heidelberg, 2000, 153, pp. 163-205.

7. Wang, Y.; Herron, N. Nanometer-sized semiconductor clusters: materials synthesis, quantum size effects, and photophysical properties. Journal of Physical Chemistry, 1991, 95, pp. 525-532.

8. Chouksey, P.; Tiwari, M.; Chandra, B.P. Luminescence Studies of Nanoparticle/Polymer Nano-Composites, International Journal of Advanced Mechanical Engineering \& Applications, 2011, 1, pp. 106-111.

9. Murray, C.B.; Noms, D.J.; Bawendi, M.G. Synthesis and Characterization of Nearly Monodisperse CdE $(E=S$, $\mathrm{Se}, \mathrm{Te}$ ) Semiconductor Nanocrystallites, Journal of the American Chemical Society, 1993, 115, pp. 8706-8715.

10. Chen, X.; Yu, Z.T.; Chen, J.S.; Yang, B. In situ hydrothermal preparation of CdS/polymer composite particles with cadmium-containing polymer latexes. Materials Letters, 2004, 58, pp. 384-386.

11. Lakowicz, J.R.; Gryczynski, I.; Gryczynski, Z.; Murphy, C.J. Luminescence Spectral Properties of CdS Nanoparticles, Journal of Physical Chemistry B, 1999, 103, pp. 7613-7620.

12. Yoon, H.; Rhym, Y.-M.; Shim, S.E. Optical properties of core/shell typed PMMA/CdS nanoparticles prepared by in situ and ex situ surfactant-free emulsion polymerization, Colloid Polymer Sciences, 2011, 289, pp. 1185-1189.

13. Sounderya, N.; Zhang, Y. Use of Core/Shell Nanoparticles for Biomedical Applications, Recent Patents on Biomedical Engineering, 2008, 1, pp. 34-42.

14. Mondal, S.P.; Mullick, H.; Lavanya, T.; Dhar, A.; Ray, S.K.; Lahiri, S.K. Optical and dielectric properties of junctionlike CdS nanocomposites embedded in polymer matrix, Journal of Applied Physics, 2007, 102(6), $064305(7 \mathrm{p})$.

15. Rong, M.Z.; Zhang, M.Q.; Liang, H.C.; Zeng, H.M. Surface derivatization of nano-CdS clusters and its effect on 
the performance of CdS quantum dots in solvents and polymeric matrices, Applied Surface Science, 2004, 228, pp. 176-190.

16. Prabhu R. R.; Abdul Khadar, M. Characterization of chemically synthesized CdS nanoparticles, PRAMANA Journal of Physics, 2005, 65, pp. 801-807.

17. Popusoi, A.; Barba, N.; Dragalina, G.; Cuculescu, E.; Caraman, M.; Robu, S. Photoluminescent polymeric nanocomposites based on aromatic chalcones derivatives (Nanocompozite polimerice fotoluminescente din derivati ai chalconelor aromatice). PRIOCHIM, 2009, Sinaia, Romania, p. 44.

18. Iovu, M.; Tiginyanu, I.; Culeac, I.; Robu, S.; Nistor, Iu.; Dragalina, G.; Enachi, M.; Petrenko, P. Nanostructured Polymer/CdS Photoluminescent Thin Films, Journal of Nanoelectronics and Optoelectronics, 2013, 7, pp. 1-5.

19. Wang, Y.; Suna, A.; Mahler, W.; Kasowski, R. PbS in polymers. From molecules to bulk solids. The Journal of Chemical Physics, 1987, 87, pp. 7315-7322.

20. Yu, W. W.; Qu, L.; Guo, W.; Peng, X. Experimental Determination of the Extinction Coefficient of CdTe, CdSe, and CdS Nanocrystals, Chemistry of Materials, 2003, 15, pp. 2854-2860.

21. Brus, L. Quantum crystallites and nonlinear optics, Applied Physics A, 1991, 53(6), pp. 465-474.

22. Chukwuocha, E. O.; Onyeaju, M. C.; Harry, T. S. T. Theoretical Studies on the Effect of Confinement on Quantum Dots Using the Brus Equation, World Journal of Condensed Matter Physics, 2012, 2, pp. 96-100.

23. Chukwuocha, E. O.; Onyeaju, M. C. Effect of Quantum Confinement on The Wavelength of CdSe, ZnS and GaAs Quantum Dots (QDs), International Journal of Scientific \& Technology Research, 2012, 1(7), pp. 21-24.

24. Zou, Y.; Li, D.; Yang, D. Noninjection Synthesis of CdS and Alloyed CdSxSe12x Nanocrystals Without Nucleation Initiators, Nanoscale Research Letters, 2010, 5, pp. 966-971.

25. Pilla, V.; Munin, E.; Dantas, N. O.; Silva A. C. A.; Andrade, A. A. Photothermal Spectroscopic Characterization in CdSe/ZnS and CdSe/CdS Quantum Dots: A Review and New Applications, Quantum Dots - A Variety of New Applications, Dr. Ameenah Al-Ahmadi (Ed.), ISBN: 978-953-51-0483-4, InTech, DOI: 10.5772/36814. http://www. intechopen.com/books/quantum-dots-a-variety-of-new-applications/photothermal-spectroscopic-characterizationin-cdse-zns-and-cdse-cds-quantum-dots-a-review-and-new-applications.

26. Murphy, C. J.; Coffer, J. L. Quantum Dots: A Primer, Applied Spectroscopy, 2002, 56(1), pp. 16A-27A.

27. Iovu, M.; Tighineanu, I.; Culeac, I.; Robu, S.; Nistor, Iu.; Dragalina, G.; Enachi, M.; Petrenko, P.; Verlan, V. Preparation and Characterization of Polymer/CdS Nanostructured Photoluminescent Films, Proceedings of the International Congress ARA37, Chisinau 4-9 June, 2013, pp. 558-561. 